\title{
DEVELOPMENT OF INTERNATIONAL KEY COMPARISONS IN THE FIELD OF CHEMICO-ANALYTICAL MEASUREMENTS
}

\author{
L. A. Konopelko, ${ }^{1}$ Yu. A. Kustikov, ${ }^{1}$ M. V. Okrepilov, ${ }^{1}$ A. V. Kolobova, ${ }^{1}$ \\ P. V. Migal, ${ }^{2}$ A. I. Krylov, ${ }^{1}$ M. S. Vonskiy, ${ }^{1}$ I. K. Chubchenko, ${ }^{1}$ \\ O. V. Efremova, ${ }^{1}$ E. V. Kulyabina, ${ }^{3}$ V. I. Dobrovolskiy, ${ }^{4}$ and A. Yu. Mikheeva ${ }^{1}$
}

UDC 543.06

\begin{abstract}
The most challenging problems in the field of chemico- and bioanalytical measurements are described. These problems are being solved, in particular, by participating in new international comparisons organized by the Consultative Committee for Amount of Substance: Metrology in Chemistry and Biology of the International Committee of Weights and Measures (CCQM CIPM). Solutions of these challenging problems are presented. The objectives and tasks of developing key comparisons in the following areas are described: isotopic measurements; determination of the purity of substances; organic analysis; bioanalytical measurements; measurements of aerosol particle parameters; measurements in the field of gas analysis and electrochemistry. It is shown that participation in international key comparisons allows obtaining valid and reliable results of measurements of the composition and properties of gas and liquid media, as well as solid substances and materials.
\end{abstract}

Keywords: comparisons, amount of substance, mole, isotope ratio, traceability, impurities, pure substances, biomarkers, counting concentration of particles, mass concentration.

Introduction. One of the main tasks of the Technical Committee (TC) 1.8 COOMET is the task of preparing the countries that are members of TC 1.8 for participation in key comparisons held by the Consultative Committee for Amount of Substance: Metrology in Chemistry and Biology (CCQM) of the International Committee of Weights and Measures (BIPM).

The amount of substance is of primary importance in chemistry and chemical metrology. It was adopted as one of the seven basic quantities of the International System of Units (SI) in 1971 by the decision of the 14th General Conference on Weights and Measures. At the same time, the basic unit of the amount of substance, the mole, was established, which was supposed to eliminate the confusion between the unit of equivalent mass (the gram-mole) and the unit of mass (the gram).

A new definition of the mole, proposed at the 106th meeting of the International Committee on Weights and Measures (CIPM) in October 2017, approved at the 26th meeting of the General Conference on Weights and Measures (CGPM) in November 2018 [1] and recommended by the International Union of Pure and Applied Chemistry IUPAC [2], is as follows:

A mole (symbol: mol) is the base unit of the amount of substance in SI. One mole contains exactly $6.02214076 \cdot 10^{23}$ structural elements. This number is the fixed numerical value of Avogadro constant, $N_{A}$, expressed in mol ${ }^{-1}$, which is called the Avogadro number.

\footnotetext{
${ }^{1}$ Mendeleev All-Russian Research Institute of Metrology (VNIIM), St. Petersburg, Russia; e-mail: 1konop1@rambler.ru.

${ }^{2}$ Ural Research Institute for Metrology (UNIIM), Branch of the Mendeleev All-Russian Research Institute of Metrology (VNIIM), Yekaterinburg, Russia; e-mail: mig@uniim.ru.

${ }^{3}$ All-Russian Research Institute of Metrological Service (VNIIMS), Moscow, Russia; e-mail: kuliabina@vniims.ru.

${ }^{4}$ Russian Metrological Institute of Technical Physics and Radio Engineering (VNIIFTRI), Mendeleevo, Moscow Region, Russia; e-mail: vid@vniiftri.ru.
}

Translated from Izmeritel'naya Tekhnika, No. 7, pp. 65-72, July, 2021. Original article submitted March 22, 2021. Accepted June 1, 2021. 
The amount of substance in a system, denoted by $n$, is a measure of the number of specific structural elements. Structural elements can be atoms, molecules, ions, electrons, other particles or certain groups of particles.

According to this definition, the amount of substance and the mass are different quantities. In addition, it eliminates duality in describing an object in terms of the amount of substance and the number of particles. Now, it is the same: the amount of substance is a measure of the number of particles.

When measuring the amount of substance and related derived quantities, such as the molar concentration or the molar content of a component, the additional uncertainty in the values of molar masses arising from the transition to the new SI is so insignificant that this transition practically does not affect the measurement uncertainty. When measuring derived quantities, which represent the ratio of these quantities, additional uncertainty does not arise at all. Therefore, there is no reason to fear that the redefinition of the mole can somehow affect the accuracy of analytical measurements, both mass measurements performed in analytical laboratories and precision measurements carried out by metrological institutes. In [3], it was emphasized that the recent revision of the SI has further escalated the need to distinguish the amount of substance from the amount counting.

The objective of this article is to investigate the importance of bio- and chemico-analytical measurements for the development of a global measurement infrastructure and international key comparisons as the only alternative to ensuring the uniformity of measurements.

Chemico-analytical measurements. A feature of modern chemico-analytical measurements is the compulsion to use off-system units: isotope ratio deltas; measurable quantities associated with protein-protein serological interactions, such as the antigen-antibody reaction, conformational measurements of the protein structure and other biologically significant indicators, currently expressed in international units of the World Health Organization; the infectious dose, the dose of the infectious agent (virions, bacteria), the size of the fragment of the specific sequence of single-strand nucleic acid, ribonucleic or deoxyribonucleic acids (RNA or DNA), etc. The decision CIPM/104-26 of 03.2015 indicates the importance of work in national metrological institutes in terms of establishing traceability of off-system units to SI units, for example, when developing absolute measurements of isotope ratios.

The most challenging task of TC 1.8 at the present stage is preparing and performing key comparisons in order to establish biologically significant parameters of macromolecules (RNA, DNA, peptides, proteins, etc.) and their complexes of biological origin (organelles, cells, etc.), as well as key comparisons of the results of the following measurements: the isotope ratios in various solid, liquid and gas substances; the content of impurities in pure solid and liquid substances; dispersed parameters of aerosols, particles, suspensions and powder materials.

Isotope measurements. The absence of high-precision isotope standards traceable to SI has led to the widespread practice of calibrating isotope measurements against fixed assumed values of certain isotope ratios or other materials arbitrarily taken as "zero points." For example, measurements of neodymium isotopes are usually calibrated against the ratio $x\left({ }^{146} \mathrm{Nd}\right) / x\left({ }^{144} \mathrm{Nd}\right)=0.7219$, taken as a constant [4]. However, this approach is not applicable to other elements, such as hydrogen or carbon, since isotope ratios are not constant for all substances. In this case, to ensure the uniformity of measurements, artefacts taken as reference materials are used. Both approaches outcome from practical necessity and manifest tacit recognition of the general need for improved isotope ratio standards.

In recent years, several cases of inappropriateness of existing reference materials for the high-precision gravimetric measurement method have been identified, which indicates the need to revise the entire system of metrological support for isotope measurements as a whole. Therefore, metrology of stable isotopes is actually just beginning to develop. The gravimetric method for the preparation of isotope reference materials was first applied 10 years ago. Nowadays many national metrological institutes use this method to prepare more reliable isotope reference materials.

Data on isotope ratios are used in almost all scientific fields: from archeometry, geochronology and geochemistry to medicine and oceanography. Scientists receive significantly important and scientifically substantiated data (for example, the age of a sample) and draw conclusions that significantly affect the development of science. Therefore, the reliability and comparability of the specified data are imperative. It can be achieved through the development of gravimetric reference materials with an assigned measurement uncertainty.

The main areas of human activity in which the analysis of stable isotopes is applied include monitoring of the environment, metrology and measuring instruments, biological sciences, forensic medical sciences, nuclear forensics. 
The most important applications of isotope analysis in the field of medicine, food industry and ecology include:

detection of the Helicobacter Pylori bacteria, which causes stomach cancer, by isotope breath test;

quality control of food products by isotope analysis;

assessment of the influence of anthropogenic processes on the content of greenhouse gases in the atmosphere by analysing isotopes in the atmospheric air.

In accordance with the decree of the BIPM [5], measurements of the isotope ratios deltas are currently traced not to the SI, but to international reference materials, which form the international delta scale for measurements of stable isotopes. However, the instability of reference materials, identified in recent years, has led to problems in the field of metrological support for measurements of stable isotopes.

Within the framework of the working group of isotope ratios of the CCQM BIPM, the following international comparisons have been organized: CCQM-K167 "Measurements of deltas of the ratios of carbon isotopes in vanillin;" CCQM-P212 "Uniformity of international reference materials for carbon isotope ratio deltas;" CCQM-P204 "CO${ }_{2}$ isotope ratios $\left(\delta^{13} \mathrm{C}\right.$ and $\left.\delta^{18} \mathrm{O}\right)$ in pure $\mathrm{CO}_{2}$;" CCQM-P213 "Delta copper isotope ratio measurements in high purity materials."

High-precision reference unit EU-1 [6] developed at VNIIM for determining the isotope composition of carbon in gas mixtures of carbon dioxide based on a cavity ringdown infrared analyser, which is a part of the State primary standard of units of molar fraction, mass fraction and mass concentration of components in gas and gas condensate environs GET 1542019, took part in all comparisons with the exception of CCQM-P213. Projects of mutually beneficial cooperation with the BIPM and the national metrological institute of Germany PTB made it possible to upgrade the EU-1 unit and achieve higher repeatability and reproducibility of measurements, as well as to publish joint scientific papers $[7,8]$.

International cooperation makes it possible to develop a common approach to ensuring the uniformity of measurements in the framework of isotope analysis and to propose optimal ways of creating a system for metrological support of measurements of stable isotopes at the national level.

Determining the purity of substances. In chemico-analytical measurements, pure substances (in solid, liquid or gas phase) are of fundamental importance. It is explained by the fact that all the results of measuring the content of any analyte in any matrix are traceable to the pure substance of this analyte. If the result of measuring the content of an analyte is to be traced to a reference material of the composition (mixture of an analyte in some matrix), then the reference material must be traced to the corresponding pure substance.

Since 2013, VNIIM and UNIIM have begun developing a methodology for the use of high-purity materials for reproducing and transferring units of quantities characterizing the chemical composition of substances and materials based on comparison standards in the form of pure chemical substances. The methodology presumes that the reference standard as a stable artefact stores the unit of the mass fraction of the main component for metals, which is reproduced on the equipment of the State primary standard of units of the mass (molar, atomic) fraction and mass (molar) concentration of the components in liquid and solid substances and materials based on coulombmetry GET 176-2019.

The main motive for the development of the methodology was the absence of agreed approaches to assessing the mass fraction of the main component by the material balance method. As noted in [9], the results of these works are consistent with international experience in characterizing pure substances based on the results of comparisons $[10,11]$ and the roadmap [12].

Within the framework of TC 1.8, comparisons in determining the purity of copper and iron have been carried out, in which the national metrological institutes of different countries participated: UNIIM, BelGIM, VNIIOFI, UME (Turkey), Ukrmetrteststandart. Comparisons have shown the consistency and compatibility of the results of measurements of the analyte mass fraction w both by high-precision direct methods and by the material balance method from an impurity composition based on the measurement equation

$$
w=100-\sum_{i=1}^{n} x_{i}-\sum_{j=1}^{r} y_{j} / 2
$$

where $x_{i}$ is the mass fraction of the $i$ th detected impurity; $n$ is the number of detected impurities; $y_{j}$ is the detection limit established for the $j$ th impurity according to the $3 \sigma$ criterion; $r$ is the number of detection limits.

As a result of the described works, the database "Comparison standards in the form of high-purity substances" (https://uniim.ru/db_etalons_ref/) was created, which includes 17 comparison standards in the form of pure metals. 
The main advantage of the material balance method is the increased accuracy of measurements and a low dependence of the measurement result on the matrix of the object under study. At that, it is important to understand the limits of applicability of such an approach to minimize systematic bias. First, for an unbiased estimate of the value of $w$, the total amount of the studied impurities should tend to the maximum possible $(\max (n+r) \rightarrow 91)$. Second, it is necessary to ensure metrological traceability of measurements of the mass fractions of at least the detected impurities $x_{i}$.

According to the practice of studies of comparison standards, the method of inductively coupled plasma mass spectrometry (ICP-MS) is suitable for determining the contents of the main number of impurities. For gas-forming elements $(\mathrm{O}, \mathrm{N}, \mathrm{H}, \mathrm{C}, \mathrm{S})$, the methods of reductive and oxidative melting are suitable. For calibration by the ICP-MS method, reference materials are usually used in the form of solutions of chemical elements, the traceability of which to GET 176-2019 can be established by titrimetric methods. For certain chemical elements, there are no reference materials with traceability to GET 176-2019. For these elements, reference materials of NIST SRM series 3100 (Standard Reference Materials. Spectrometry, Single Element Standard Solutions, https:/www.nist.gov/srmors/viewTable.cfm?tableid=39) or reference materials of foreign production with established traceability to NIST SRM reference materials are used.

Solving the problems of ensuring metrological traceability of composition measurements in the field of inorganic chemical analysis is a task of gradual approximation, i.e., in conditions of impossibility to ensure measurements of the analyte mass fraction for a number of chemical elements by direct absolute methods, it is necessary to involve indirect methods.

At present, it is possible to assess the purity of the comparison standard by the material balance method for $91 \mathrm{impu}-$ rities: 5 gas-forming elements; 72 elements determined by ICP-MS method; 14 elements in the form of theoretical estimates for noble gases and radioactive elements. The next approximation is the development of the classification of comparison standards (bringing the number to 73 separate elements). Then it is necessary to provide a closed cycle, in which the impurity composition of each reference standard will be determined through other reference standards, so that each refinement of the impurity composition of one reference standard leads to a refinement of the mass fraction of the analyte of other reference standards. Such an iterative approach is to be implemented in the next works on the basis of the collection of pure substances, which is stored in the UNIIM and has 76 positions of 64 separate elements. This collection does not include elements of the platinum group (Ru, Rh, Pd, Os, Ir, Pt, Au), as well as the elements that do not have stable isotopes (U, Th).

Thus, the accumulated experience allows setting initial approximations that are close enough to the truth, and subsequent iterations can significantly reduce the uncertainty of knowledge about the real purity of a substance and thereby ensure metrological traceability of measurements in this area.

Organic analysis. In organic analysis, traceability to SI is formed on the basis of pure organic substances, thoroughly analytically studied and metrologically characterized in detail. It is the mass fraction of the main component in a pure organic substance that provides traceability to one of the basic SI units - the amount of substance (mole), in the case of organic analysis - to a mole of a certain organic substance.

Thus, the first link in forming a chain of metrological traceability in the field of organic analysis is the determination of the purity of organic substance. The generally accepted international approach to determining the purity of organic substances consists in the maximum possible study of the material in order to confirm its identity and determine all possible impurity components. After that, the mass fraction of the main component is calculated by the indirect mass balance method as the difference: $100 \%$ minus the sum of impurities. It is better to use at least two analytical methods to measure the same quantity. In general, the mass balance method for organic substances involves the mandatory measurement of impurity components of four groups: impurities of related compounds, in general case - components that can be measured by the same analytical method as the main substance, most often, its isomers and (or) congeners;

impurities of free and crystallization water;

impurities of volatile organic compounds, for example, residual organic solvents after purification of the basic substance by recrystallization;

impurities of non-volatile compounds with a potential contribution of organic non-volatile components and inorganic compounds (for example, metals in any form).

To obtain additional data confirming the integral characteristics of pure organic substances, direct methods of coulombmetry, cryometry, and differential scanning calorimetry are used. 
Equipment included in the State primary standard of units of mass (molar) fraction and mass (molar) concentration of organic components in liquid and solid substances and materials based on liquid and gas chromatography-mass spectrometry with isotope dilution and gravimetry GET 208-2019 allows fully implementing the methods of mass balance and differential scanning calorimetry. GET 208-2019 is formed on the basis of serial analytical instruments commercially available in the foreign and domestic markets. The uniqueness of GET 208-2019 is not the instrumental design, but the knowledge of the characteristics and fine-tuning of the equipment, as well as the developed analytical and metrological procedures included in the GET 208-2019 in the form of reference documentation and allowing achieving the highest measurement accuracy in organic analysis.

Over the period from 1998 to the present, the Russian Federation has taken part in more than 35 international comparisons organized by the CCQM BIPM, which cover the most different aspects of organic analysis, including the determination of the purity of organic substances belonging to different chemical groups (aldrin, L-valine, folic acid, bisphenol A, dimethyl sulfone) [13]. Information on the approved calibration and measuring capabilities (CMC) based on the results of comparisons is publicly available on the official website of the BIPM.

Bioanalytical measurements. Bioanalytical measurements are the youngest and fastest growing section of chemico-analytical measurements. Since 2017, the CCQM BIPM, which was previously called the Consultative Committee for Metrology in Chemistry, is called the Committee for Metrology in Chemistry and Biology. Progress in science and technology and the development of bioinformatics have led to a breakthrough in measuring the processes occurring in living matter, and, above all, in the human body. Throughout the history of the 166 Nobel Prizes in chemistry, 73 prizes were awarded for discoveries in the field of bioanalysis and catalytic processes in the human body.

For more than 10 years, TC 1.8 has been actively involved in the work of CCQM in the field of the development of a global metrological infrastructure for bioanalytical measurements.

In 2015-2019, pilot and key comparisons were carried out: CCQM-P137 (pilot comparisons in measurement of the catalytic activity of $\alpha$-amylase in blood serum); CCQM-P164 (pilot comparisons (two rounds) in measurement of the mass fraction of growth hormone in blood serum); CCQM-K86, CCQM-K86.b, CCQM-K86.c (key comparisons aimed at confirming CMC lines of national metrological institutes in the field of measuring the content of genetically modified organisms (GMOs)).

In 2021, key comparisons CCQM-K86.d are planned to confirm the CMC lines of the institutes for measurements of DNA in a matrix rich in proteins - to determine DNA biomarkers for the presence of pork in beef.

In 2018-2020, pilot comparisons CCQM-P199 and P199.b were carried out in measurements of the copy number concentration of genomic sequences of the human immunodeficiency virus HIV-1 and the SARS-CoV-2 coronavirus. Within the framework of these comparisons, pilot laboratories prepared highly concentrated samples of pure RNA, the measurements of which were performed by methods "orthogonal" to the dPCR method: mass spectrometry with isotope dilution and flow fluorimetry of single molecules. The results of measurements by the "orthogonal" methods were in good agreement with the results obtained using the dPCR method. In 2021, the key comparisons CCQM-K176 began in measurements of variation in the copy number of the HER2 genomic sequence, a biomarker of breast cancer. Comparisons are planned in measurements of the level of DNA methylation as an important prognostic marker of the development of oncopathologies.

The main tasks to be solved in the field of protein analysis include the development and validation of reference methods for assessing the purity of peptides and proteins, qualitative and quantitative analysis of peptides and proteins in complex biological matrices and biopharmaceuticals, measurements of the catalytic activity of enzymes, determining specific characteristics of biotherapeutic products and antibodies.

The youngest field of bioanalysis is cell analysis. It includes the identification and measurement of intact cells number and their properties, reflecting specific cellular functions in complex matrices and mixtures. The main characteristics of a cell include viability, size, morphology, features of localization in space (growth on the surface or in suspension), the area occupied on the surface, the presentation of certain antigens on the cell surface, and the expression of biomarkers. At that, the approaches developed within the framework of cell analysis should be applicable to both eukaryotic and prokaryotic cell cultures. Although microbiological methods for assessing the content of microorganisms have a long history, the application of metrological approaches to these types of measurements is accompanied by a number of problems. Thus, the creation of reference materials using living cells with biological activity and certain physicochemical properties clearly requires the 
development of new methodologies. Particular hopes in this area of cell analysis are associated with the use of synthetic biology. Areas of cell analysis that require special attention in the near future include measurements of the morphology and characteristics of the cell phenotype, measurements of indicators of the cell biological activity and the characteristics of intracellular signalling networks [14].

The comparisons carried out to date and being planned are concentrated on the quantification of cells attached to the surface (CCQM-P123, CCQM-P197) and in suspension (CCQM-P205, CCQM-P217). At this point, the main successes in this area are mainly associated with studies of the metrological characteristics of cell quantification methods, which can be performed both automatically, using flow cytometry, and in manual mode, using a hemocytometer (Goryaev's chamber). The results of measurements by volumetric flow cytometry are directly traceable to SI units. However, the selection and preparation (staining) of the sample, the cytometer calibration, the measurement mode optimization and the processing of the results obtained contribute to the measurement error, the minimization of which is still an unsolved problem.

One of the promising ways to create reference materials for cell measurements is the synthesis of latex particles that mimic the size and shape of a cell, with the possibility of integrating fluorescent dyes that simulate staining with fluorophore-labelled antibodies. The start of the first pilot CCQM comparisons in order to assess the cell quantification methods using synthetic latexes is scheduled for 2021 .

Measurements of parameters of aerosol particles. In recent years, experts involved in environmental monitoring and indoor and outdoor air quality control have been faced with the task of obtaining reliable information on the parameters and characteristics of aerosol particles. These indicators include optical and chemical properties, size and shape distribution of particles, mass concentration of particles and particle charge, content of elemental and organic carbon, anions and cations, basic metals (lead, arsenic, cadmium, mercury, nickel).

In this regard, the first pilot and key comparisons in this measurement category are planned. At VNIIFTRI, the State Primary Standard of dispersed parameters of aerosols, suspensions and powdered materials GET 163-2020 has been created, which ensures the reproduction, storage and transfer of units of particle size, counting and mass concentration of particles, as well as the zeta potential of particles, and allows solving critical tasks in the designated areas.

Today the leading metrological institutes of such countries-participants of TC 1.8 COOMET, as Germany, China, and Belarus, have such reference systems. The use of the new instrumental base will allow TC 1.8 to take an active part in CCQM works and increase the levels of reliability and traceability of the results of measuring the parameters of aerosol particles to the SI system.

Gas and electrochemical measurements. The issues and tasks of the development of key comparisons in the new measurement categories, organized by CCQM BIPM, considered in the article, do not mean a decrease in the activity of TC 1.8 in other "traditional" measurement categories, such as "Gas analysis," "Electrochemistry," etc. National metrological institutes of Ukraine, Belarus, Kazakhstan, and Russia participate in the key comparisons in the categories "Gas Analysis" and "Electrochemistry." Improvement of state primary standards allows these institutes to expand their participation in new key comparisons, planned in accordance with the strategies of the corresponding CCQM working groups on gas and electrochemical analyses.

VNIIM has created systems and facilities for verification and certification of reference gas mixtures based on NO, $\mathrm{NO}_{2}, \mathrm{SO}_{2}, \mathrm{H}_{2} \mathrm{~S}, \mathrm{NH}_{3}$, prepared by the gravimetric method, and has investigated them and included in GET 154-2019 [15]. They are designed to measure the following characteristics:

molar fraction of the main impurities $\mathrm{O}_{2}, \mathrm{CO}, \mathrm{CO}_{2}, \mathrm{CH}_{4}, \mathrm{~N}_{2}, \mathrm{H}_{2}, \mathrm{Ar}$ in high-purity gases $\mathrm{He}, \mathrm{N}_{2}, \mathrm{Ar}, \mathrm{O}_{2}$;

molar fraction, mass fraction and mass concentration of ethanol in aqueous solutions and gas mixtures;

molar fraction of methanol in hydrocarbon gas mixtures;

molar fraction of ${ }^{12} \mathrm{CO}_{2},{ }^{13} \mathrm{CO}_{2}$, the isotope ratios in gas mixtures (and for certification of comparison standards for the composition of gas mixtures containing ${ }^{12} \mathrm{CO}_{2},{ }^{13} \mathrm{CO}_{2}$, in high pressure cylinders);

mass concentration of components of pre-explosive concentrations of vapours of combustible and explosive liquids in the range of $5-50 \%$ of the lower concentration limit of flame propagation.

The characteristics of GET 154-2019 allow VNIIM to actively participate in the comparisons planned in the CCQM BIPM strategy in the field of gas analysis for the following measurement objects: greenhouse gases; ozone and active gases at 
atmospheric level; natural gas; refinery gases; volatile organic compounds in nitrogen and air; forensic medical gases; binary and multicomponent gas mixtures corresponding to the composition of emissions; pure gases with impurity analysis; gravimetric gas mixtures; dynamically prepared gas mixtures; medical gases.

The creation of standards in the field of electrochemical measurements in the national metrological institutes of the countries participating in TC 1.8 COOMET (Russia, Ukraine, Moldova, Kazakhstan, Belarus) allowed organizing a number of pilot and key comparisons in the measurement category "Electrochemistry."

To verify the equivalence of national standards in the field of $\mathrm{pH}$-metry, three international pilot comparisons have been carried out using a phosphate buffer solution with $\mathrm{pH} \approx 7.00$ : COOMET 322/RU/04 (2004) "Pilot comparisons of buffer solutions based on potassium dihydrogen phosphate $\left(\mathrm{KH}_{2} \mathrm{PO}_{4}\right)+$ sodium hydrogen phosphate $\left(\mathrm{Na}_{2} \mathrm{HPO}_{4}\right)$ at temperatures of 15, 25, and 37 ${ }^{\circ}$ C;" COOMET 421/RU/08 (2008) "Additional comparisons of working pH standards;" COOMET 655/RU$\mathrm{a} / 15$ (2015) "Pilot comparisons in determining the $\mathrm{pH}$ value of phosphate buffer $\sim 7.00 . "$

In the nearest future, it is planned to carry out comparisons in other ranges of the $\mathrm{pH}$ scale. Thus, for 2023 , a key comparison in determining the $\mathrm{pH}$ value of a tetraoxalate buffer solution with a $\mathrm{pH} \approx 1.65$ is planned.

In the field of specific electrical conductivity measurements, the following comparisons have been carried out: COOMET 361/RU-a/06 (2006) "Pilot comparisons in the field of electrical conductivity measurements;" CCQM-K36.2016 (2017) "Electrolytic conductivity at $0.5 \mathrm{~S}^{-1}$ and $5 \mathrm{mS}^{-1}$;" EURAMET CCQM-SC EURAMET.QM-S12 (2019) "EURAMET comparison 1462."

Based on the use of coulombmetric standards of units of mass (molar, atomic) fraction and mass (molar) concentration of components in liquid and solid substances and materials, pilot comparisons have been carried out:

COOMET 520/RU/1 "Pilot comparisons in the field of measuring the concentration of hydrogen ions in a hydrochloric acid solution (mol/kg of the solution)," participants: Russia (UNIIM), Ukraine (Ukrmetrteststandart);

COOMET 645/RU/14 "Pilot comparisons in the field of measuring the mass fraction of copper and impurities in oxygen-free copper rod of KM6 M0016 grade in order to determine the copper purity," participants: Belarus (BelGIM), Russia (VNIIOFI, UNIIM), Turkey (UME), Ukraine (Ukrmetrteststandart);

COOMET 672/RU/5 "Pilot comparisons in the field of measuring the mass fraction of iron in pure iron," participants: Belarus (BelGIM), Moldova (NIM), Russia (VNIIFTRI, VNIIOFI, UNIIM), Ukraine (Ukrmetrteststandart).

Conclusion. The development of international infrastructure in the field of bio- and chemico-analytical measurements is determined by the need to ensure global comparability of measurement results when solving problems of sustainable development and control of climate change, green power engineering and healthcare. As a result of participation of metrological institutes of countries participating in TC 1.8 COOMET in international key comparisons, the new reference systems and the following methods have been developed and applied: measurements of isotope ratios in solid, liquid and gas substances; establishing biologically significant parameters of macromolecules (RNA, DNA, peptides, proteins, etc.) and their complexes of biological origin (organelles, cells, etc.); measurements of the content of impurities in pure solid and liquid substances; measurements of dispersed parameters of aerosols, particles, suspensions and powder materials.

New types of reference materials, which provide the calibration capabilities of the countries participating in TC 1.8, have been created. Participation in key comparisons allows harmonizing approaches to calculating the uncertainty of measurement results, contributes to obtaining valid and reliable results of measurements of the composition and properties of gas and liquid substances, as well as solid substances and materials.

\section{REFERENCES}

1. General Conference for Weights and Measures (CGPM), Resolution 1 of 26 meeting of CGPM, Nov. 13-16, 2018, https://www.bipm.org/en/committees/cg/cgpm/26-2018/resolution-1, acc. 06.23.21.

2. R. Marquardt, J. Meija, Z. Mester, et al., "Definition of the mole (IUPAC Recommendation 2017)," Pure Appl. Chem., 90, No. 1, 175-180 (2018).

3. R.J.C. Brown and P.J.Brewer, “What is a mole?” Metrologia, 57, No. 6,065002(2020), https://doi.org/10.1088/16817575/ab9db7. 
4. J. R. de Laeter, J. K. Böhlke, P. de Bièvre, et al., Atomic Weights of the Elements. Review 2000, IUPAC Technical Report, De Gruyter (2013), https://doi.org/10.1351/pac200375060683.

5. International Committee for Weights and Measures, Proceedings of Session I of the 104th Meeting, March 9-10, 2015, https://www.bipm.org/utils/en/pdf/CIPM/CIPM2015-I-EN.pdf, acc. 11.13.2018.

6. L. A. Konopelko, Y. K. Chubchenko, and V. V. Beloborodov, Int. Conf. Laser Optics (ICLO) (2018), pp. 288-288, https://doi.org/10.1109/LO.2018.8435389.

7. J. Viallon et al., Metrologia, 57, No. 5, 055004 (2020), https://doi.org/10.1088/1681-7575/ab948c.

8. E. Flores et al., "Comparison of isotope ratio measurement capabilities for $\mathrm{CO}_{2}$ : Sample preparation and characterization by Isotope Ratio Infrared Spectroscopy," EGU General Assembly Conference Abstracts (2020), p. 8518.

9. L. A. Konopelko, P. V. Migal, and E. P. Sobina, "Development of transfer measurement standards in the form of high-purity metals," Stand. Obraztsy, 15, No. 2, 15-24 (2019).

10. J. Vogl, H. Kipphardt, H.-J. Heinrich, et al., CCQM-P107.1 Purity of Zinc with Respect to Six Defined Metallic Analytes, Draft Report, BAM Federal Institute for Materials Research and Testing, Berlin (2014).

11. J. Vogl et al., CCQM-P149 Purity Determination of Zinc to be Used as Primary Standard for Zinc Determination, Draft B, BAM Federal Institute for Materials Research and Testing, Berlin (2016).

12. J. Vogl, CCQM-IAWG17-28 Roadmap for the Purity Determination of Pure Metallic Elements. Basic Principles and Helpful Advice (2017), https://www.bipm.org/wg/CCQM/IAWG/Allowed/April_2017/CCQM-IAWG17-28.pdf, acc. Feb. 3 .2021.

13. A. Yu. Mikheeva and A. I. Krylov, "Traceability in organic analysis. Report 1: Equivalence of the national and international measurement standards," Etalony, Stand. Obraztsy, 16, No. 3, 5-21 (2020).

14. N. Faruqui, A. Kummrow, B. Fu, et al., Front. Bioeng. Biotechnol., 7, 456 (2020).

15. A. V. Kolobova, L. A. Konopelko, and O. G. Popov, "State primary standard of units of molar part, mass part and mass concentration of components in gas and gas condensate environs GET 154-2019," Etalony, Stand. Obraztsy, 16, No. 3, 23-35 (2020), https://doi.org/10.20915/2687-0886-2020-16-3-23-35. 